\title{
Implications of inappropriate prescription of oral nutritional supplements on the quality of life of cancer outpatients: a cross-sectional comparative study
}

\author{
Islam A. Hassanin ${ }^{1}$ (D) Reem F. M. Salih ${ }^{1} \cdot$ Marwa H. M. Fathy $^{1} \cdot$ Eman A. Hassanin $^{1} \cdot$ Dina H. Selim $^{1,2} \mathbb{D}$
}

Received: 24 September 2021 / Accepted: 13 January 2022 / Published online: 25 January 2022

(c) The Author(s) 2022

\begin{abstract}
Background and aims Oral nutritional supplements (ONS) are considered a cornerstone in the treatment plan of malnutrition in cancer patients. However, the prevalence of inappropriate prescription of ONS is high. In this study, we aim to investigate the effect of inappropriate oral nutritional supplementation (consisting of prescription of ONS without evident clinical indication, or the absence of ONS when at risk of malnutrition) on the quality of life of cancer outpatients.

Methods A cross-sectional comparative study was conducted in 104 cancer outpatients, receiving ONS without prior malnutrition risk screening $(n=51)$, and patients not receiving ONS $(n=53)$. Nutritional risk screening was performed using the abridged patient-generated subjective global assessment (ab-PG-SGA). The quality of life was assessed using EORTC QLQ-C30 version 3.0 questionnaire. Multivariate analysis was conducted to determine the predictors of quality-of-life scales. Age (years), malnutrition (ab-PG-SGA scores), BMI ( $\left.\mathrm{kg} / \mathrm{m}^{2}\right)$, TSF $(\mathrm{mm}), \operatorname{MUAC}(\mathrm{cm})$, ONS (yes, no) were entered into the linear regression analysis as predictors (backward stepwise linear regression analysis).

Results The prevalence of malnutrition risk (ab-PG-SGA $\geq 6$ ) was $74 \%$. The median score of the ab-PG-SGA for ONS receiving group was significantly higher $(p=0.045)$. Furthermore, univariate analysis showed that the scores of the global health status (QoL) and the role functioning (RF) scales were significantly lower for the ONS receiving group $(p=0.020$ and $p=0.016$, respectively). Multivariately, malnutrition, inappropriate ONS prescription, and triceps skin fold were found to be predictors of the RF scale, while malnutrition was the only predictor for the QoL.

Conclusion The inappropriate ONS prescription does not improve nutritional status or quality of life of cancer outpatients.
\end{abstract}

Keywords Oral nutritional supplements $\cdot$ Cancer $\cdot$ Malnutrition $\cdot$ Quality of life $\cdot$ Screening $\cdot$ Role functioning

\section{Introduction}

Malnutrition is considered a prognostic factor, which affects cancer patients [1]. The prevalence of malnutrition in cancer patients varies considerably from 15 to $80 \%$ [2]. Furthermore, the rate of mortality based on tumor-related malnutrition ranges between 20 and 30\% [3]. Malnutrition possesses

Reem F. M. Salih and Marwa H. M. Fathy are contributed equally in this work.

Dina H. Selim

selim.dina@yahoo.com

1 Faculty of Pharmacy, Alexandria University, Alexandria 21521, Egypt

2 Educational and Medical Complex, Al Mawasah University Hospital, Alexandria University, Alexandria 21526, Egypt a negative impact on the quality of life of cancer patients [4, 5]. It is demonstrated that malnutrition is an independent predictor of poor quality of life in cancer patients on several levels: physical, functional, emotional, and social $[5,6]$. For instance, malnutrition was correlated with poor physical functioning in oral or oropharyngeal cancer patients[7]. Thus, the European Society for Clinical Nutrition and Metabolism (ESPEN) recommends malnutrition screening, as well as nutritional intervention for malnourished cancer patients or patients at risk of malnutrition [3]. Nutritional screening can be implemented using non-invasive tools, e.g., the abridged patient-generated subjective global assessment (ab-PG-SGA). This tool is derived from the PG-SGA developed by Ottery (1996) [8]. It was found that the ab-PG-SGA is a valid tool for detection of malnutrition risk in cancer outpatients at a score $\geq 6$ [9]. 
The intervention procedures recommended by ESPEN guidelines consist of nutritional counseling as well as adding oral nutritional supplementation, to increase the oral intake by cancer patients [3]. Oral nutritional supplements (ONS) are homogenous mixtures of balanced nutrients for enhanced caloric intake, commercially available for oral administration $[10,11]$. The general criteria for ONS prescription encompass (1) nutritional risk screening or nutritional assessment; (2) identification of the possible etiology of malnutrition; (3) describing the goals of ONS prescription and establishing the desired outcome. These processes should be followed by monitoring and further assessment of the ONS current use, combined with dietary advice $[12,13]$. ONS were found to effectively increase the oral intake and to enhance the quality of life in cancer patients [14-17]. However, the prevalence of inappropriate prescription of ONS seems to be high (30-70\%) [18-21]. On the other hand, the inappropriate prescription of ONS may fall into three categories: over-prescription, mis-prescription, and under-prescription $[12,22]$. Over-prescription involves prescription of ONS without evident clinical indication, while mis-prescription includes overdoses or clinically irrelevant extended duration of treatment of ONS. Finally, under-prescription is manifested as the absence of ONS therapy with evident clinical indication[22]. Furthermore, assessment of ONS intake is significant and challenging. One study concluded that the compliance of cancer patients was low for ONS intake, and therefore, it may affect patients' weight loss. Hence, it was suggested that education strategies should be implemented for patients receiving ONS [23].

The relationships between inappropriate ONS prescription, the prevalence of malnutrition, and quality of life in cancer outpatients are under-studied. To our knowledge, our study is the first, aiming to investigate the relationship between the inappropriate prescription of ONS, nutritional status, and the quality of life of cancer outpatients.

\section{Patients and methods}

\section{Patients}

A single-centered cross-sectional comparative study was conducted to compare the effect of inappropriate ONS prescription pattern in two groups (receiving or not receiving ONS). Two groups were included in this study:

Group 1: Patients inappropriately prescribed ONS (without malnutrition risk screening prior to inclusion in the study).

Group 2: Patients who did not receive any nutritional support.

All cancer outpatients ( $\geq 18$ years, able to communicate, answer the study questions, and tolerate measurements) at Ayade El-Mostaqbal Hospital were approached to participate in this study in the period between February and April 2021. Patients who were on enteral or parenteral nutritional support were excluded.

\section{Outcome measures}

\section{Demographics and patterns of ONS prescription}

Demographic data of the patients including age and gender were collected by the researcher through patients' interviews. Other medical data, e.g., type of cancer, and biochemical tests' results were collected from the hospital records, retrospectively.

To identify the patterns of ONS prescription, a face-toface interview with the patients was conducted to assess the nutritional status and a "Yes/No" response on whether they were prescribed any nutritional supplement (NS). When applicable, we identified a pattern of prescription of ONS: non-selective to nutritional status (disease-related malnutrition, i.e., no nutritional assessment) and without a dietitian referral, prior to inclusion to the study. Rather, other medical reasons were reported, e.g., loss of appetite, frailty, aging, and the preference of the patient. In our study, this prescription pattern was referred to as "Inappropriate ONS." The ONS prescribed by the physician prior to this study was either a powdered form or a drink form, with variable contents. The prescription was based on either forms or both in conjunction.

\section{Nutritional screening and anthropometric measurements}

Nutritional screening and anthropometric measurements were conducted following the initiation of the study. To identify patients at risk of malnutrition, the abridged patient-generated subjective global assessment (ab-PGSGA) was utilized. The ab-PG-SGA is considered an applicable screening tool in the oncological outpatient setting. The ab-PG-SGA is a derivative of the PG-SGA tool, including the initial four questions of the PG-SGA with a total score ranging from 0 to 35 . Higher scores indicate risk of malnutrition. An ab-PG-SGA score of $\geq 6$ was considered to be at risk of malnutrition [9]. In addition, anthropometric measurements, including mid-upper arm circumference (MUAC) measured using a measuring tape, and triceps skin fold (TSF) measured using a TSF caliber on the posterior surface of the arm, were collected. Body mass index (BMI) was calculated as the weight in kilograms divided by the square of height in meters. 


\section{Quality of life}

EORTC QLQ-C30 version 3.0 questionnaire developed by the European Organization for Research and Treatment of Cancer (EORTC) was used to assess the quality of life and compare the group non-selectively receiving ONS and the patients who did not receive any NS. The EORTC QLQ-C30 version 3.0 questionnaire is composed of 30 items: five functional scales, the global quality-of-life (QoL) scale, three symptom scales, and six single items of symptoms commonly reported by oncological patients. The scores were linearly transformed ranging from 0 to 100 . Higher scores on the global QoL and the functional scales represent a higher quality of life and higher functioning level, respectively. On the other hand, lower scores on the symptom scale or symptom items represent a lower level of the reported symptoms [24].

\section{Sample size}

A convenient sample size (104 cancer outpatients) was obtained based on the prevalence of the administration of oral nutritional supplements (ONS) in cancer outpatients. Based on a literature search of previous studies, the prevalence of oncological patients receiving ONS was $31.8 \%$, while oncological patients not receiving any nutritional support (NS) was estimated to be $60.2 \%$ [25]. The sample size estimation for this cross-sectional comparative study design was based on an effect size (Cohen's $h$ ) of $0.578,95 \%$ confidence level (two sided, $\alpha / P=0.05, \beta$ level $=0.80$, and considering $10 \%$ of the patients to drop-out of the study), yielding a requirement of minimum 49 patients in each group [26, 27].

\section{Statistical analysis}

The analysis of the collected data was conducted using Statistical Package for Social Sciences (SPSS, Inc., Chicago, IL, USA) version 23.0. Data are presented as percentages and mean \pm standard deviation. Independent samples $t$ test was used to compare the continuous variables between the two groups (ONS and non-ONS receiving patients). Alternatively, the Mann-Whitney $U$ test was implemented to compare between groups in the case of non-normally distributed data. Pearson's chi-squared test was used to compare groups for categorical variables. Scores of the EORTC QLQ-C30 scales and items were compared between ONS and non-ONS receiving patients using the Mann-Whitney $U$ test.

Linear regression analysis was conducted to investigate the correlation between malnutrition risk and the components (scales and items) of the EORTC QLQ-C30. Multivariate regression analysis was conducted to investigate the potential predictors of the EORTC QLQ-C30 scales, which relates significantly to the inappropriate use of ONS. Multivariately, age (years), malnutrition (ab-PGSGA scores), BMI ( $\left.\mathrm{kg} / \mathrm{m}^{2}\right)$, TSF (mm), MUAC (cm), ONS (yes, no) were entered into the linear regression analysis as predictors (backward stepwise linear regression analysis), where entry and removal criteria were $p<0.05$ and $p>0.10$, respectively. A statistically significant result was set at $P$-value $\leq 0.05$ level (two-tailed).

\section{Results}

\section{Patients' characteristics}

Table 1 shows the baseline characteristics and demographics of the included oncology outpatients. In our study, based on the ab-PG-SGA (score $\geq 6$ ), the prevalence of oncological patients identified at risk of malnutrition was $74 \%$. The patients were $52.7 \pm 12.3$ years, with the majority of the patients being females $(67.3 \%)$. Most of the cohort were breast cancer patients $(33.7 \%)$. The patients were stratified according to the history of ONS use. Patients receiving ONS showed more risk to malnutrition than patients who do not receive any ONS, with a higher median score for ONS receiving group $(p=0.045$; Table 1).

\section{Quality of life}

Univariate analysis of the quality of life indicated that the score of QoL of the ONS receiving group $(43.9 \pm 16.8)$ was significantly $(p=0.020)$ lower than of the non-ONS receiving group (50.6 \pm 14.6$)$. Additionally, the role functioning scale showed a similar trend with a lower score for ONS receiving group ( $p=0.016$; Table 2$)$. On the other hand, there were no significant differences between the two groups in the other components of the EORTC QLQC30. Notably, malnutrition risk (ab-PG-SGA score $\geq 6$ ) was significantly correlated with all the components of the quality of life in our cohort, except for the symptom items (diarrhea and constipation) (presented in Table 2).

Multivariate analysis was conducted to determine the predictors of QoL and the role functioning scale (RF) scores. Malnutrition $(p<0.0005)$, the inappropriate use of ONS ( $p=0.037)$, and triceps skin fold $(p=0.002)$ were significantly related to role functioning. Non-significant predictors were age, BMI, and MUAC. However, only malnutrition was significantly related to $\mathrm{QoL}(p=0.006$; Table 3). Age, BMI, TSF, MUAC, and inappropriate ONS use were non-significant as predictors of QoL. 
Table 1 Baseline characteristics of 104 cancer patients

\begin{tabular}{|c|c|c|c|}
\hline Variable & Non-ONS & ONS & $P$ value \\
\hline$N(\%)$ & $53(51 \%)$ & $51(49 \%)$ & \\
\hline Gender, $n(\%)$ & & & 0.836 \\
\hline Males & $18(33.9 \%)$ & $16(31.4 \%)$ & \\
\hline Females & $35(66.0 \%)$ & $35(68.6 \%)$ & \\
\hline Age $($ mean $\pm S D)$, years & $50.9 \pm 11.07$ & $54.5 \pm 13.4$ & 0.092 \\
\hline \multicolumn{4}{|l|}{ Type of cancer, $n(\%)$} \\
\hline Bladder & $0(0 \%)$ & $2(3.9 \%)$ & \\
\hline Bone & $1(1.9 \%)$ & $4(7.8 \%)$ & \\
\hline Breast & $22(41.5 \%)$ & $13(25.5 \%)$ & \\
\hline Cervical & $1(1.9 \%)$ & $0(0 \%)$ & \\
\hline Endometrial & $0(0 \%)$ & $3(5.9 \%)$ & \\
\hline Gastrointestinal & $14(26.4 \%)$ & $13(25.5 \%)$ & \\
\hline Glioma & $0(0 \%)$ & $1(2 \%)$ & \\
\hline Lung & $7(13.2 \%)$ & $3(5.9 \%)$ & \\
\hline Lymphoma & $3(5.7 \%)$ & $3(5.9 \%)$ & \\
\hline Ovarian & $3(5.7 \%)$ & $4(7.8 \%)$ & \\
\hline Prostate & $0(0 \%)$ & $3(5.9 \%)$ & \\
\hline Uterine & $2(3.8 \%)$ & $2(3.9 \%)$ & \\
\hline Body mass index $\left(\mathrm{kg} / \mathrm{m}^{2}\right)$ & $29.95 \pm 6.52$ & $28.6 \pm 6.1$ & 0.294 \\
\hline TSF (mm) & $26.2 \pm 4.92$ & $24.9 \pm 4.1$ & 0.226 \\
\hline $\operatorname{MUAC}(\mathrm{cm})$ & $34.9 \pm 4.5$ & $33.2 \pm 4.1$ & 0.085 \\
\hline \multicolumn{4}{|c|}{ Screening tool scores, median (range) } \\
\hline ab-PG-SGA & $8(0.0-17.0)$ & $10(0.0-22.0)$ & $0.045^{*}$ \\
\hline \multicolumn{4}{|c|}{ Laboratory markers $($ mean $\pm \mathrm{SD})$} \\
\hline ALT (IU/L) & $29.9 \pm 31.2$ & $20.5 \pm 18.7$ & $0.016^{*}$ \\
\hline AST (IU/L) & $28.4 \pm 17.2$ & $20.9 \pm 17.7$ & $0.015^{*}$ \\
\hline ANC $\left(\right.$ cells $\left./ \mathrm{mm}^{3}\right)$ & $6342.2 \pm 9402.6$ & $3728.5 \pm 3394.2$ & 0.098 \\
\hline Bilirubin (mg/dL) & $0.76 \pm 0.81$ & $0.55 \pm 0.85$ & $0.018 *$ \\
\hline Creatinine $(\mathrm{mg} / \mathrm{dL})$ & $0.90 \pm 1.25$ & $1.06 \pm 1.89$ & 0.376 \\
\hline Platelet count $\left(\times 10^{3} / \mu \mathrm{L}\right)$ & $253.9 \pm 102.5$ & $278.7 \pm 385.6$ & 0.312 \\
\hline
\end{tabular}

$T S F$, triceps skin fold; $M U A C$, mid-upper arm circumference; $a b-P G-S G A$, abridged patient-generated subjective global assessment; $A L T$, alanine aminotransferase; $A S T$, aspartate aminotransferase; $A N C$, absolute neutrophil count; ONS, oral nutrition supplementation receiving patients; non-ONS, patients receiving no ONS

*Statistically significant result

\section{Discussion}

The results of this study indicated that the prevalence of malnutrition risk in cancer outpatients, based on the abPG-SGA, was 74\%, which is in line with previously published reports. Silva et al. reported that malnutrition was identified in $71 \%$ of cancer patients using PG-SGA [28]. The high prevalence of tumor-related malnutrition reflects poor nutritional status following inappropriate prescription of ONS. Although the mean score of the ab-PG-SGA for patients receiving ONS in our cohort was significantly higher, patients not receiving ONS were presented with an ab-PG-SGA median score $>6$, indicating malnutrition risk (Table 1). These results reflect the inappropriate pattern of
ONS prescription, which is presented with a combination with under-prescription and over-prescription.

Nutritional intervention, consisting of a dietary advice alone or in combination with an ONS, is considered an important milestone in the treatment of disease-related malnutrition. However, ONS prescription is often complicated with heterogenous reasons, which may compromise its effectiveness, e.g., disregarding nutritional status [12]. It was reported that the pattern of inappropriate ONS prescription is as high as 75\% [18]. In our study, we identified an over-prescription, as well as an under-prescription process. The most reported causes for ONS prescription were frailty, aging, and loss of appetite. Moreover, in some circumstances, patients asked for an ONS prescription, in order to increase nutritional intake. Our findings were consistent 
Table 2 The EORTC QLQ-C30 quality of life in oncological patients inappropriately receiving ONS or not receiving ONS and its correlation with malnutrition risk
Table 3 Stepwise backward multivariate linear regression analysis showing the predictors of the EORTC QLQ-C30 scales in oncological outpatients

\begin{tabular}{llllll}
\hline EORTC QLQ-C30 scale/item & Non-ONS & ONS & & Malnutrition risk & \\
\hline & $\begin{array}{l}\text { Mean } \pm \text { SD } \\
(n=53)\end{array}$ & $\begin{array}{l}\text { Mean } \pm \text { SD } \\
(n=51)\end{array}$ & $P$ value & Standardized $\beta$ & $P$ value \\
Global health status $($ QOL) & & & & & \\
$\quad$ Global health status (QOL) & $50.6 \pm 14.6$ & $43.9 \pm 16.8$ & $0.020^{*}$ & -0.268 & $0.006^{*}$ \\
Functional scale & & & & & $<0.005^{*}$ \\
Physical functioning (PF) & $48.5 \pm 21.9$ & $49.1 \pm 21.4$ & 0.753 & -0.441 & $<0.005^{*}$ \\
Role functioning (RF) & $57.9 \pm 28.0$ & $44.4 \pm 27.6$ & $0.016^{*}$ & -0.397 & $<0.005^{*}$ \\
Emotional functioning (EF) & $44.9 \pm 28.8$ & $51.5 \pm 28.4$ & 0.198 & -0.367 & $<0.005^{*}$ \\
Cognitive functioning (CF) & $58.8 \pm 29.3$ & $57.2 \pm 31.3$ & 0.817 & -0.441 & $<0.005^{*}$ \\
Social functioning (SF) & $72.6 \pm 30.2$ & $63.4 \pm 30.2$ & 0.119 & -0.367 & $<0.005^{*}$ \\
Symptoms scale & & & & & $<0.005^{*}$ \\
Fatigue (FA) & $49.5 \pm 25.7$ & $53.2 \pm 25.4$ & 0.501 & 0.484 & $<0.005^{*}$ \\
Nausea and vomiting (NV) & $30.2 \pm 28.3$ & $28.1 \pm 26.8$ & 0.760 & 0.463 & $<0.005^{*}$ \\
Pain (PA) & $50.3 \pm 28.6$ & $53.3 \pm 24.8$ & 0.698 & 0.414 & $0.003^{*}$ \\
Dyspnea (DY) & $59.7 \pm 34.8$ & $54.2 \pm 29.8$ & 0.304 & 0.386 & $<0.005^{*}$ \\
Insomnia (SL) & $58.3 \pm 37.8$ & $47.1 \pm 30.7$ & 0.086 & 0.288 & 0.213 \\
Appetite loss (AP) & $41.5 \pm 33.3$ & $46.7 \pm 28.6$ & 0.421 & 0.598 & 0.070 \\
Constipation (CO) & $27.0 \pm 31.4$ & $37.3 \pm 33.1$ & 0.106 & 0.123 & $0.001^{*}$ \\
Diarrhea (DI) & $21.4 \pm 30.7$ & $23.5 \pm 33.5$ & 0.819 & 0.178 & 0.326 \\
Financial difficulties (FI) & $30.2 \pm 32.9$ & $41.2 \pm 29.5$ & 0.059 & & \\
\hline
\end{tabular}

*Statistically significant result

${ }^{\dagger}$ Analyzed by the Mann-Whitney $U$ test

\begin{tabular}{llllc}
\hline EORTC QLQ-C30 scale/predictor & Unstandardized $\boldsymbol{B}$ & SE $B$ & $95 \%$ CI & $P$ value \\
\hline Role functioning scale (RF) & & & & \\
$\quad$ Malnutrition & -2.413 & 0.537 & -3.479 to -1.348 & $<0.0005^{*}$ \\
$\quad$ Inappropriate use of ONS & -10.716 & 5.062 & -20.761 to -0.671 & $0.037^{*}$ \\
$\quad$ Triceps skin fold & -1.728 & 0.556 & -2.831 to -0.626 & $0.002^{*}$ \\
$\quad$ Constant & 122.849 & 16.089 & $90.926-154.772$ & $<0.0005^{*}$ \\
Global health status (QoL) & & & & \\
$\quad \begin{array}{l}\text { Malnutrition } \\
\text { Constant }\end{array}$ & -0.902 & 0.323 & -1.543 to -0.261 & $0.006^{*}$ \\
\end{tabular}

*Statistically significant result with the study published by P. Dominguez Castro et al. reporting that about one-third of the general practitioners involved in the prescription of ONS for cancer patients were not adherent to the prescription criteria, and that malnutrition was not prioritized in the prescription process (no nutritional risk screening was conducted) [12]. Therefore, our aim was to evaluate the effect of this prescription pattern on the overall quality of life in cancer outpatients. Despite the positive effect reported previously for ONS on the quality of life of cancer patients [16, 29], our results showed contrary effect in the case of inappropriate ONS prescription. The mean of the QoL scale was lower in ONS receiving group compared to non-ONS receiving group (Table 2). The role functioning scale (RF) was also significantly lower for ONS receiving group ( $p=0.016)$. Especially, RF scale possesses a prognostic effect on the overall survival in cancer patients [30]. Malnutrition, inappropriate use of ONS, and TSF were predictors of the RF scale. The inappropriate ONS prescription was negatively related to the RF scale $(B=-10.710$; $p=0.037)$. In this regard, the European Society for Clinical Nutrition and Metabolism (ESPEN) guidelines recommend that the prescription of ONS is subjected to cancer patients who are diagnosed as malnourished or at risk of malnutrition. However, the guidelines stated that it was still unclear whether ONS prescription without a dietary counseling could provide an improved clinical outcome [3]. A recently published trial found that the inappropriate ONS prescription alone or in combination with dietary advice did not 
improve the survival in cancer patients [31]. Additionally, Uster et al. concluded that nutritional intervention including nutritional counseling and oral nutritional supplementation successfully improved energy and protein intake with no improvement of nutritional statuses or quality of life, related to advanced cancer stages [32]. Stemming from our results, we can also infer that the prescription of ONS alone without a dietary advice did not provide any positive effect on the quality of life of cancer outpatients. Although the emotional scale showed an improvement in ONS-treated group, it was not statistically significant.

Interestingly, malnutrition risk was significantly correlated with all the functional scales, as well as symptoms items, except for diarrhea and constipation. These results were similar to previous studies, stating that malnutrition risk is an independent predictor of lower QoL [5]. Subsequently, malnutrition risk was the only predictor of the QoL in our study $(B=-0.902 ; p=0.006)$. Several reports have pointed out the negative influence of malnutrition on the quality of life [4], response to treatment [33], complications, and costs [34]. Malnutrition was also found to impact the overall survival in cancer patients [35].

The limitation of the study is manifested in the cross-sectional study design. This design did not allow the quantification of clinical outcome measures. Additionally, the results of the study should be interpreted cautiously. On the other hand, the cross-sectional design offered a sufficient resort to address the conception of the study, and the comparison between groups.

In summary, this study provided insights on the high prevalence of malnutrition in cancer outpatients and the negative influence of inappropriate ONS prescription on the quality of life in cancer patients. The inappropriate use of ONS did not provide any improvements on the quality of life in cancer patients. We recommend the implementation of the general criteria for prescription of ONS for cancer patients, which was included by P. Dominguez Castro et al. [12]. The necessity of dietary advice along with ONS prescription, as well as nutritional follow-up, is considered a major issue to be investigated in future research. Also, it is necessary to raise the awareness of the prescribing the physicians about nutritional risk screening and assessment procedures. Subsequently, it will be imperative to refer patients at risk of malnutrition to a "dietitian" for further assessment and setting a treatment plan.

\section{Conclusion}

The inappropriate pattern of ONS prescription exhibited in cancer outpatient setting does not seem to improve nutritional statuses or quality of life of cancer patients, and the prevalence of malnutrition risk remains high.
Acknowledgements We would like to thank the patients for the participation in our study. Also, we would like to thank the hospital board of directors at Ayade El-Mostaqbal Hospital for the facilitation of conducting this study. Finally, we would like to thank PharmD board of supervisors at the Faculty of Pharmacy, Alexandria University.

Author contribution Islam A. Hassanin: methodology, formal analysis, writing —original draft, writing — review and editing. Reem F. M. Salih and Marwa H. M.: data curation, methodology. Eman A. Hassanin: formal analysis, writing — review and editing. Dina H. Selim: conceptualization, methodology, supervision, writing—review and editing.

Funding Open access funding provided by The Science, Technology \& Innovation Funding Authority (STDF) in cooperation with The Egyptian Knowledge Bank (EKB).

Availability of data and material Data is available, and it will be provided upon request to the corresponding author.

Code availability Not applicable.

\section{Declarations}

Ethics approval The study protocol was approved by the Ethics Committee, Faculty of Medicine, Alexandria University (IRB NO.: 00012098).

Consent to participate The patients included were informed about the anonymity of the collected data and signed an informed consent to approve participation in this study. To identify the patterns of ONS prescription, a signed consent was obtained from the hospital board of directors at Ayade El-Mostaqbal Hospital.

Consent for publication Not applicable.

Conflict of interest The authors declare no competing interests.

Non-financial interest. Authors Reem F. M. Salih and Marwa H. M. Fathy participated in this study as part of the PharmD program, supervised at Faculty of Pharmacy, Alexandria University, Alexandria 21521, Egypt.

Open Access This article is licensed under a Creative Commons Attribution 4.0 International License, which permits use, sharing, adaptation, distribution and reproduction in any medium or format, as long as you give appropriate credit to the original author(s) and the source, provide a link to the Creative Commons licence, and indicate if changes were made. The images or other third party material in this article are included in the article's Creative Commons licence, unless indicated otherwise in a credit line to the material. If material is not included in the article's Creative Commons licence and your intended use is not permitted by statutory regulation or exceeds the permitted use, you will need to obtain permission directly from the copyright holder. To view a copy of this licence, visit http://creativecommons.org/licenses/by/4.0/.

\section{References}

1. Sonneborn-Papakostopoulos M et al (2021) Quality of life, symptoms and dietary habits in oncology outpatients with malnutrition: a cross-sectional study. Med Oncol 38(2):20 
2. von Haehling S, Anker SD (2010) Cachexia as a major underestimated and unmet medical need: facts and numbers. J Cachexia Sarcopenia Muscle 1(1):1-5

3. Arends $J$ et al (2017) ESPEN guidelines on nutrition in cancer patients. Clin Nutr 36(1):11-48

4. Lis CG et al (2012) Role of nutritional status in predicting quality of life outcomes in cancer-a systematic review of the epidemiological literature. Nutr J 11:27

5. Polański J, Jankowska-Polańska B, Mazur G (2021) Relationship between nutritional status and quality of life in patients with lung cancer. Cancer Manag Res 13:1407-1416

6. Rios TC et al (2021) A poorer nutritional status impacts quality of life in a sample population of elderly cancer patients. Health Qual Life Outcomes 19(1):90

7. Jager-Wittenaar $\mathrm{H}$ et al (2011) Malnutrition and quality of life in patients treated for oral or oropharyngeal cancer. Head Neck 33(4):490-496

8. Jager-Wittenaar H, Ottery FD (2017) Assessing nutritional status in cancer: role of the Patient-Generated Subjective Global Assessment. Curr Opin Clin Nutr Metab Care 20(5):322-329

9. Gabrielson DK et al (2013) Use of an abridged scored PatientGenerated Subjective Global Assessment (abPG-SGA) as a nutritional screening tool for cancer patients in an outpatient setting. Nutr Cancer 65(2):234-239

10. Kim SH et al (2019) The effect of nutrition intervention with oral nutritional supplements on pancreatic and bile duct cancer patients undergoing chemotherapy. Nutrients 11(5):1145

11. Stratton RJ, Elia M (2010) Encouraging appropriate, evidencebased use of oral nutritional supplements. Proc Nutr Soc 69(4):477-487

12. Dominguez Castro $P$ et al (2020) General practitioners' views on malnutrition management and oral nutritional supplementation prescription in the community: a qualitative study. Clin Nutr ESPEN 36:116-127

13. Cudjoe A (2013) Guide to prescribing adult oral nutritional supplements (ONS) in the community. Wandsworth Clinical Commissioning Group, London

14. Barber MD et al (1999) The effect of an oral nutritional supplement enriched with fish oil on weight-loss in patients with pancreatic cancer. Br J Cancer 81(1):80-86

15. Bauer $J$ et al (2005) Compliance with nutrition prescription improves outcomes in patients with unresectable pancreatic cancer. Clin Nutr 24(6):998-1004

16. Baldwin $\mathrm{C}$ et al (2012) Oral nutritional interventions in malnourished patients with cancer: a systematic review and meta-analysis. J Natl Cancer Inst 104(5):371-385

17. van der Meij BS et al (2012) Oral nutritional supplements containing n-3 polyunsaturated fatty acids affect quality of life and functional status in lung cancer patients during multimodality treatment: an RCT. Eur J Clin Nutr 66(3):399-404

18. Ragubeer R, Patel HJ (2012) Pilot to improve the appropriate prescription of oral nutritional supplements within the Walsall area. Proc Nutri Soc 70(OCE5):E272

19. Gall MJ, Harmer JE, Wanstall HJ (2001) Prescribing of oral nutritional supplements in Primary Care: can guidelines supported by education improve prescribing practice? Clin Nutr 20(6):511-515

20. Kennelly S et al (2009) The use of oral nutritional supplements in an Irish community setting. J Hum Nutr Diet 22(6):511-520
21. Cummins P, Thomson M (2015) A dietetic prescribing support initiative leads to significant cost saving efficiencies, by improving the clinical management of oral nutritional supplement prescribing in primary care. Clin Nutr ESPEN 10(5):e204

22. Cadogan CA et al (2020) A systematic scoping review of interventions to improve appropriate prescribing of oral nutritional supplements in primary care. Clin Nutr 39(3):654-663

23. Wan G-Y et al (2021) Assessment of compliance with oral nutritional supplementation and exploration of barriers and facilitators for patients after gastrectomy: a mixed-methods study. J Adv Nurs 77(6):2845-2859

24. Aaronson NK et al (1993) The European Organization for Research and Treatment of Cancer QLQ-C30: a quality-of-life instrument for use in international clinical trials in oncology. $\mathrm{J}$ Natl Cancer Inst 85(5):365-376

25. Hébuterne $X$ et al (2014) Prevalence of malnutrition and current use of nutrition support in patients with cancer. J Parenter Enter Nutr 38(2):196-204

26. Sharma $\mathrm{S}$ et al (2019) How to calculate sample size for observational and experiential nursing research studies? Natl J Physiol Pharm Pharmacol 2019:1

27. Casagrande JT, Pike MC (1978) An improved approximate formula for calculating sample sizes for comparing two binomial distributions. Biometrics 34(3):483-486

28. Silva FRDM et al (2015) Factors associated with malnutrition in hospitalized cancer patients: a croos-sectional study. Nutri J 14(1): 123

29. de van der Schueren MAE et al (2018) Systematic review and meta-analysis of the evidence for oral nutritional intervention on nutritional and clinical outcomes during chemo(radio)therapy: current evidence and guidance for design of future trials. Ann Oncol 29(5):1141-1153

30. Meier A et al (2015) Role functioning is associated with survival in patients with hepatocellular carcinoma. Qual Life Res 24(7):1669-1675

31. Söderström L et al (2020) Dietary advice and oral nutritional supplements do not increase survival in older malnourished adults: a multicentre randomised controlled trial. Ups J Med Sci 125(3):240-249

32. Uster A et al (2013) Influence of a nutritional intervention on dietary intake and quality of life in cancer patients: a randomized controlled trial. Nutrition 29(11):1342-1349

33. Ovesen L, Hannibal J, Mortensen EL (1993) The interrelationship of weight loss, dietary intake, and quality of life in ambulatory patients with cancer of the lung, breast, and ovary. Nutr Cancer 19(2):159-167

34. Correia MI, Waitzberg DL (2003) The impact of malnutrition on morbidity, mortality, length of hospital stay and costs evaluated through a multivariate model analysis. Clin Nutr 22(3):235-239

35. Zhang $X$ et al (2019) Malnutrition and overall survival in older adults with cancer: a systematic review and meta-analysis. J Geriatr Oncol 10(6):874-883

Publisher's Note Springer Nature remains neutral with regard to jurisdictional claims in published maps and institutional affiliations. 